\title{
Utilizing Disease-Specific Organ Shape Components for Disease Discrimination: Application to Discrimination of Chronic Liver Disease from CT Data
}

\author{
Dipti Prasad Mukherjee ${ }^{1,2}$, Keisuke Higashiura ${ }^{3}$, Toshiyuki Okada ${ }^{2}$, Masatoshi Hori ${ }^{2}$, \\ Yen-Wei Chen ${ }^{3}$, Noriyuki Tomiyama ${ }^{2}$, and Yoshinobu Sato ${ }^{2}$ \\ ${ }^{1}$ Indian Statistical Institute, Kolkata, West Bengal, India \\ ${ }^{2}$ Department of Radiology, Graduate School of Medicine, Osaka University, Japan \\ ${ }^{3}$ Graduate School of Information Science and Engineering, Ritsumeikan University, Japan
}

\begin{abstract}
We describe a method to capture disease-specific components in organ shapes. A statistical shape model, constructed by the principal component analysis (PCA) of organ shapes, is used to define the subspace representing inter-subject shape variability. The first PCA is applied to the datasets of healthy organ shapes to define the subspace of normal variability. Then, the datasets of diseased shapes are projected onto the orthogonal complement (OC) of the subspace of normal variability, and the second PCA is applied to the projected datasets to derive the subspace representing the disease-specific variability. To calculate the OC of an n-dimensional subspace, a novel closed-form formulation is developed. Experiments were performed to show that the support vector machine classification in the OC subspace better discriminated healthy and diseased liver shapes using 99 CT data. The effects of the number of training data and the difference in segmentation methods on the classification accuracy were evaluated to clarify the characteristics of the proposed method.
\end{abstract}

Keywords: Statistical shape model, orthogonal complement, support vector machine, liver fibrosis, computer-aided diagnosis.

\section{Introduction}

Discriminating diseased organs from healthy ones based on the variations of organ shapes is one of the important goals of computational anatomy. The early structural change in cortical grey matter caused by Alzheimer's disease is a well-studied problem [1]. This work studied the shape variations based on the local geometric measurement from an average representation of the shape. However, global deformation patterns may be more important for studying some pathological anatomies such as chronic liver disease (CLD). To our knowledge, the development of computational tools for modeling disease-specific global deformation patterns is still insufficient.

To study the variability of normal anatomy or pathological structures caused by the disease, the statistical shape model (SSM), which is constructed by the principal component analysis (PCA) of organ shapes, is one of the basic representation schemes [2]. 
However, it would be ideal if it was possible to eliminate the normal variability of organ shapes from the variability of diseased organs to effectively study diseasespecific behavior.

We propose to use the disease-specific shape component SSM that objectively eliminates the variability of healthy shapes. We generate an SSM with a given set of healthy organ shapes and then design a subspace that is the orthogonal complement (OC) of the healthy shape SSM. The projection of a new healthy shape sample into the OC subspace is ideally expected to result in sufficiently small components. Subsequently, the projections of diseased shapes into this OC subspace are expected to mostly consist of the disease-specific components after eliminating the normal variability. In our literature survey, the OC subspace was utilized for content-based image retrieval [3]. However, it has not been applied to discriminate subtle variations in anatomical shapes caused by the disease, such as those addressed by Golland et al. [4]. Here, we do not assume that the components of shape changes caused by the disease are in the OC subspace, but assume and demonstrate that using the diseasespecific components in the OC subspace is useful for disease discrimination.

In this study, we address the problems of shape-based discrimination of CLD. With respect to computer-assisted diagnosis (CAD) of CLD, the local shape has been studied [5]. However, its usefulness is limited to the discrimination of serious stages, where bumpy contours of the liver surface are clearly observed. Although the radiologists' observations of the global changes of CLD are typically used in clinical diagnosis, the modeling of global liver shape changes in CAD has been insufficient. Because these shape change patterns are often subtle, their detection may be a difficult task even for experienced radiologists. The purpose of this work is to objectify and quantify these shape changes by statistical shape analysis, and in particular, the shape components specific to CLD using the OC subspace. We eventually aim to develop a CT-based imaging biomarker for staging liver fibrosis.

The main contributions of this study are as follows: (1) We have shown that the anatomical difference of shape is clarified in the OC space, which improves the support vector machine (SVM) classification accuracy. (2) We combine the OC-based SVM classifier with automated CT segmentation of the liver to evaluate its performance and limitations in clinical use. (3) In addition, a complete closed-form solution of estimation of n-dimensional OC space is provided from the methodological point of view.

\section{Methodology}

SSM provides a family of shape vectors, $\mathbf{x} \in \mathbb{R}^{n}, \mathbf{x}=\overline{\mathbf{x}}+\mathbf{P} b$, where $\overline{\mathbf{x}}$ represents the mean shape and $\mathbf{P}$ represents the eigenvector of some shape space samples, $\boldsymbol{\chi}$. The SSM parameter $b$ is varied within $\pm \kappa \sigma, \sigma=\sqrt{\lambda}$, where $\lambda$ represents the eigenvalue of the SSM space $\chi$. In general, the $\kappa$ value of 2 or 3 is chosen and the probability of the parameter $b$ is assumed to follow a Gaussian distribution. Given the sample shape $\mathrm{S}$, which is not included in $\chi$, the estimation of $b$ could be a multidimensional optimization of the distance $\|\mathbf{S}-\mathbf{x}(b)\|$. This distance could be either 
Euclidean or Mahalanobis distance between the point clouds $\mathrm{S}$ and $x$ for a given $b$. However, in case the new shape $\mathbf{S} \notin \chi$ is already registered with the family $\boldsymbol{\chi}$, then $b=\mathbf{P}^{\mathrm{T}}(\mathbf{S}-\overline{\mathbf{x}})$, where ${ }^{\mathrm{T}}$ represents the transpose. If $\boldsymbol{\chi}$ represents a healthy or disease class of organs, the SSM parameter $b$ can be estimated using $b_{\mathrm{N}}=\mathbf{P}_{\mathrm{N}}^{\mathrm{T}}\left(\mathbf{x}_{\mathrm{N}}-\overline{\mathbf{x}}_{\mathrm{N}}\right)$, where subscript $\mathrm{N}$ represents a healthy organ class. Assuming that a global deformation of the organ shape caused by a specific disease is represented by the eigenvectors $\mathbf{P}_{\mathrm{D}}$, the disease shape $\mathbf{x}_{\mathrm{D}}$ is represented as

$$
\mathbf{x}_{\mathrm{D}}=\overline{\mathbf{x}}_{\mathrm{N}}+\mathbf{P}_{\mathrm{N}} b_{\mathrm{N}}+\mathbf{P}_{\mathrm{D}} c_{\mathrm{D}}
$$

where $c_{\mathrm{D}}$ is the SSM parameter. Let $\mathbf{P}_{\mathrm{OCN}}$ be the sub-components of $\mathbf{P}_{\mathrm{D}}$ projected onto the OC of the healthy SSM. Thus, we can modify Eq. (1) as,

$$
\mathbf{x}_{\mathrm{D}}=\overline{\mathbf{x}}_{\mathrm{N}}+\mathbf{P}_{\mathrm{N}} b_{\mathrm{N}}^{\prime}+\mathbf{P}_{\mathrm{OCN}} c_{\mathrm{OCN}} .
$$

$\mathbf{P}_{\mathrm{OCN}}$ is calculated as the principal component (PC) of the OC-transformed disease samples $\mathbf{x}_{\mathrm{D}}$ that is projected onto the OC of the healthy SSM. Therefore, to create the OC-transformed disease-specific SSM, we obtain $c_{\mathrm{OCN}}$ by $c_{\mathrm{OCN}}=\mathbf{P}_{\mathrm{OCN}}^{\mathrm{T}}\left(\mathbf{x}_{\mathrm{D}}-\right.$ $\left.\overline{\mathbf{x}}_{\mathrm{N}}-\mathbf{P}_{\mathrm{N}} b_{\mathrm{N}}^{\prime}\right)$. Next, we present a complete closed-form solution for the estimation of $n$-dimensional OC space.

\subsection{Orthogonal Complement of Principal Components}

The OC of a subspace $M \subset \mathbb{R}^{n}$ is given by $M^{\perp}=\left\{w \in \mathbb{R}^{n}: w^{T} v=0, \forall v \in M\right\}$. $M^{\perp}$ represents a subspace and $\mathbb{R}^{n}=M \oplus M^{\perp}$. If $\operatorname{dim} M=q$, then $\operatorname{dim} M^{\perp}=n-q$. We are interested in finding the OC of a subset of PCs of a covariance matrix. The OC of a correlation matrix is used for tracking principal and/or minor subspaces for applications in signal processing involving time series data [6]. In that case, the OCs weigh the correlation matrix in each iteration of tracking. In our case, the orthogonal complement of a few leading PCs defining healthy organ shape will be used to project the disease specific shape.

Assuming that $n$-D shape space is split into the $r$ and $p$-D subspaces, such that the first $p$ PCs of the shape space are chosen to calculate the OC and $\boldsymbol{\phi}$ is $(r+p) \times p$ eigenvectors of a covariance matrix $\mathbf{Z}((r+p) \times(r+p)$ dimension), we can decompose $\boldsymbol{\phi}$ into two matrices as,

$$
\boldsymbol{\phi}^{\mathrm{T}}=\left[\boldsymbol{\alpha}^{\mathrm{T}} \boldsymbol{\beta}^{\mathrm{T}}\right]
$$

where, $\boldsymbol{\alpha}$ represents the $(r \times p)$ matrix containing the first $r$ rows of $\boldsymbol{\phi}$ and $\boldsymbol{\beta}$ represents the $(p \times p)$ matrix containing p last rows of $\boldsymbol{\phi}$. To find out the OC of the p-dimensional dominant subspace, we need to do orthogonal decomposition of $\boldsymbol{\beta}$. Three promising orthogonal decompositions are the QR decomposition, SVD decomposition, and polar decomposition. The most stable and suitable way is to use polar decomposition, where factors are unique and coordinate-independent [7]. Using polar decomposition, $\boldsymbol{\beta}=\boldsymbol{\theta} \mathbf{U}$, where $\mathbf{U}$ represents the positive definite and $\boldsymbol{\theta}$ represents 
the orthogonal matrix. The orthogonal factor $\boldsymbol{\theta}$ represents the closest possible orthogonal matrix to $\boldsymbol{\beta}$ [7]. The physical interpretation of polar decomposition is that the orthogonal factor $\boldsymbol{\theta}$ provides a rotation and the positive definite factor $U$ provides a stretch along the basis vectors defined in $U$.

Therefore, $(r \times p)$ upper sub-matrix $\boldsymbol{\alpha}$ of $\boldsymbol{\phi}$ has to be rotated along the orthogonal part of the remaining lower $(p \times p)$ sub-matrix $\boldsymbol{\beta}$ of $\boldsymbol{\phi}$. Therefore, we define $\mathbf{f}_{1}=\boldsymbol{\alpha} \boldsymbol{\theta}^{\mathrm{T}}$ and $\mathbf{f}_{2}=\mathbf{f}_{1}(\mathbf{I}+\mathbf{U})^{-1}$. Note that $(\mathbf{I}+\mathbf{U})$ is always positive definite. Then, the $\mathrm{OC}$ of $p$ dimensional subspace of $\boldsymbol{\phi}$ is given by,

$$
\mathbf{O}=\left[\begin{array}{c}
\mathbf{I}_{\mathbf{r}}-\mathbf{f}_{1} \mathbf{f}_{2}^{\mathrm{T}} \\
-\mathbf{f}_{1}^{\mathrm{T}}
\end{array}\right] .
$$

The matrices $\mathbf{f}_{1}$ and $\mathbf{f}_{2}$ are of dimensions $(r \times p)$, so $\mathbf{O}$ is $(r+p) \times r$ dimensional orthonormal matrix. In Eq. (4) the lower $(p \times r)$ part of $\mathbf{O}$ represents the orthogonal rotation of the upper $(r \times p)$ part of $\boldsymbol{\phi}$ in the direction orthonormal to the lower $(p \times p)$ part of $\boldsymbol{\phi}$. The $\left[(\mathbf{I}+\mathbf{U})^{-1}\right]\left[\left(\boldsymbol{\alpha} \boldsymbol{\theta}^{\mathrm{T}}\right)^{\mathrm{T}}\right]$ part of the right hand side of the upper $(r \times p)$ part of $\mathbf{0}$ represents the pseudo-inverse of $\left[\boldsymbol{\alpha}^{\mathrm{T}}\right]$ and acts as OC projector from the theory of least square. It can be easily shown that $\mathbf{O}^{\mathrm{T}} \mathbf{O}=\mathbf{I}$. Therefore, $\mathbf{O}$ represents the $\mathrm{OC}$ of the $p$ dimensional subspace defined by the p PCs of the covariance matrix $\mathbf{Z}$. Then, because $\boldsymbol{\phi}$ is also an orthogonal matrix, $\mathbf{0}^{\mathrm{T}} \boldsymbol{\phi}=\mathbf{0}$.

Therefore, by taking the first $p$ eigenvectors of the healthy organ subspace $\mathbf{P}_{\mathrm{N}}$, Eq. (4) yields the OC of the healthy organ subspace. Next, we define the diseased organ subspace in the OC of the healthy shape.

\subsection{Diseased Organ Subspace}

The generation of healthy shape subspace is straightforward and provides the basis vectors $f_{1}, f_{2}, \ldots, f_{k}$, of normal variations of the $k$ dimensional healthy subspace. For an $n$ dimensional shape sample $\mathbf{x}_{\mathrm{N}}$, we define the $(n-k)$ dimensional subspace as the OC of the healthy subspace. The OC is calculated using Eq. (4). Let the basis vectors of the OC be $o_{1}, o_{2}, \ldots, o_{n-k}$. We project each of the $n$ dimensional vectors $\left(\mathbf{x}_{\mathrm{D}}-\overline{\mathbf{x}}_{\mathrm{N}}\right)$ of the disease datasets to the $(n-k)$ dimensional OC subspace. The projected vectors are given by

$$
\mathbf{x}_{\mathrm{D}}^{\prime}=\sum_{i=1}^{(n-k)}\left\langle\left(\mathbf{x}_{\mathrm{D}}-\overline{\mathbf{x}}_{\mathrm{N}}\right), o_{i}\right\rangle o_{i}
$$

Then, PCA is performed on the projected $(n-k)$ vectors $\mathbf{x}_{\mathrm{D}}^{\prime}$. The first l basis vectors of this projected disease data set provides the disease specific subspace, $g_{1}^{\prime}, g_{2}^{\prime}$, $g_{3}^{\prime}, \ldots, \quad g_{l}^{\prime}$. Note that $g_{i}=\sum_{j=1}^{(n-k)}\left\langle g_{\mathrm{j}}^{\prime}, e_{j}\right\rangle o_{j}$, where $e_{j}$ denotes a $(n-k)$ dimensional unit vector whose $j$-th element is one and others are zero. Therefore, we obtain the disease subspace $g_{1}, g_{2}, g_{3}, \ldots, g_{l}$, where all the basis vectors are orthogonal to each other and to the healthy subspace. 


\section{Experimental Results}

\subsection{Dataset and Experimental Methods}

We evaluated the conventional and proposed methods using 99 (53 healthy and 46 diseased) liver shape data. All the shape data represented as polygon models are registered to have the same topology of polygon models. The method for correspondence among the shape data was as follows. One reference data was initially selected, to which all other data were non-rigidly registered [8] to obtain the average shape. All the data were then registered to the average shape to obtain the final correspondence. The liver shape and size was normalized using the standard space defined by an approximation of the abdominal cavity according to a previous report [9]. The conventional method used SSM constructed from the mixed datasets of healthy and diseased liver shapes [4], whereas the proposed method used the OC-transformed diseasespecific SSM described in Section 2. The SSM parameters were used for SVM classification of healthy and disease. SVM classification using the conventional method is basically the same as the method described by Golland et al. [4].

The diseased liver shapes were segmented from contrast-enhanced CT data taken from 46 CLD patients. Among them, the livers of 29 patients were histologically diagnosed (through biopsy or tissue resection) to be fibrotic, i.e., stage F1 or higher according to the established criteria [10], and 17 patients underwent minimally invasive liver therapy. The healthy liver shapes were derived from the CT data of 53 potential liver donors. All the data were retrospective. Figure 1 shows typical healthy and diseased liver shapes.

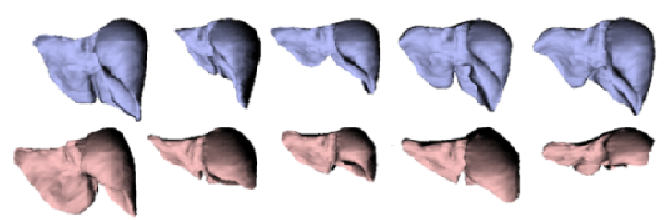

Fig. 1. Examples of liver shapes. Top (blue): Healthy livers. Bottom (red): Diseased livers.

We used two types of CT segmentation methods: manual tracing and fully automated. The average accuracy of automated segmentation in the Jaccard index was 0.897 for healthy and 0.867 for diseased livers. We evaluated the results under three segmentation conditions: (1) both training and test data were manually traced ("Bothmanual"); (2) the training data were manually traced but the test data were automated ("Training-manual \& test-automated"); and (3) both were automated ("Bothautomated"). The first condition is unrealistic in a clinical setting because manual segmentation for each patient is a large burden for the clinical staff. The second condition will be clinically feasible because each patient data is automatically processed once the classifier has been trained. The third condition is also clinically feasible and has an advantage that automated addition of the training data is possible in the clinical routine. 
We performed $k$-fold cross validation. Only within the training data, we further performed another (leave-one-out) cross validation to select the optimal parameter values involved in the classifier so that the highest classification accuracy was attained. Note that the test data were totally separated from the classifier training as well as the parameter optimization. In the parameter optimization, the number of PCs was optimized. In the disease-specific SSM, the number of PCs in the healthy SSM, whose OC was used, was also optimized. We used a linear SVM and no parameter was involved in it. A Gaussian-RBF SVM was not used because its lower performance in our problem was confirmed via a preliminary cross-validation study. The above $k$-fold cross validation was performed for 30 different $k$ partitions of the data, which were randomly generated, and the results were averaged.

\subsection{Results}

We plotted the ROC curves by shifting the discrimination hyper-plane of SVM along its normal for the proposed and conventional methods [4]. We also evaluated the classification accuracy that is defined as the ratio of the numbers of correctly classified shapes to all the shapes when the SVM hyper-plane is not shifted.

Figure 2 shows the ROC curves and classification accuracy under the three segmentation conditions, when $k=15$, i.e., 15 -fold cross-validation was performed. The number of PCs was approximately 37 in the conventional method, whereas it was 8 for the healthy SSM and 20 for the OC-transformed disease-specific SSM in the proposed method. The proposed method showed higher performance on ROC and classification accuracy under the conditions "Both-manual" and "Both-automated" than the conventional method. Under "Training-manual \& testing-automated," the classification accuracy was the worst. The reduction of the accuracy from "Both-manual" to "Both-automated" was larger in the proposed method than in the conventional method, although the accuracy was still better in "Both-automated." Overall, the proposed method shows better performance, even though a larger accuracy reduction was observed when the automated segmentation was used.

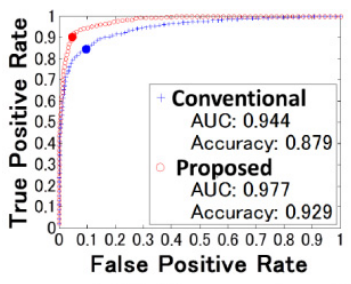

(a) Both-manual

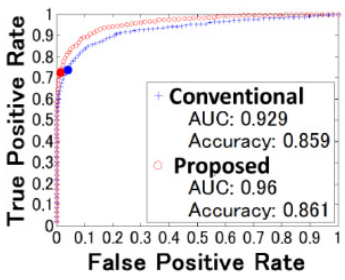

(b) Training-manual \& test-automated

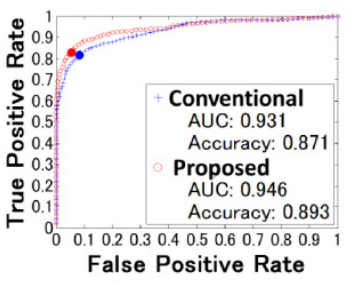

(c) Both-automated

Fig. 2. ROC curves. Conventional: Blue, Proposed: Red. The knots on the curves denote the classification results when the discrimination plane was not shifted, i.e., they represent original SVM classification results. AUC represents the area under the curve. 
Figure 3 shows the effects of the number of training data on the classification accuracy using different $k$ values in $k$-fold cross-validation. The number of training data was 50, 66, and 92 for $k=2,3$, and 15 , respectively. The accuracy improvement was large in the proposed method for increased number of training data compared with the conventional method.

Figure 4 visualizes the shape deformation patterns corresponding to the normal of the SVM hyper-plane (See captions of Fig. 4 for the details). As in Fig. 4(b), the proposed method showed more subtle shape deformation for the discrimination than the conventional method.

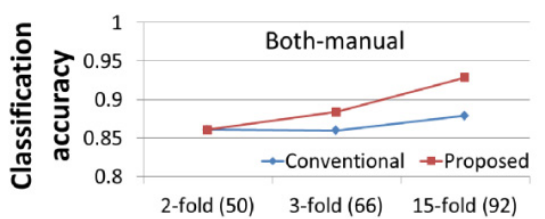

(a) Both-manual

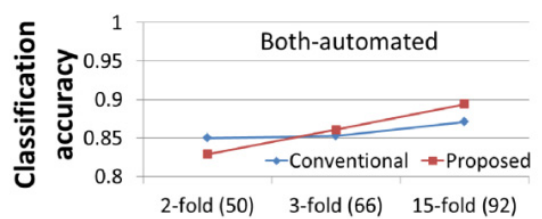

(b) Both-automated

Fig. 3. Effects of number of training data on classification accuracy (Conventional: Blue, Proposed: Red). The number of training data is shown in parenthesis along the horizontal axis.

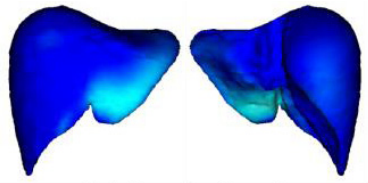

(a) Conventional

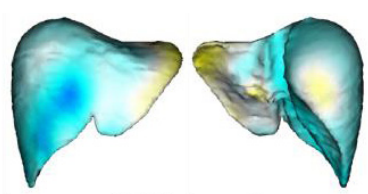

(b) Proposed

Fig. 4. Deformation patterns corresponding to the normal vector of the discrimination hyperplane of SVM. The liver shape is the projection of the average shape onto the discrimination plane. The normal vector originates from the discrimination plane and heads to the disease side. Blue and cyan represent large and slight atrophy (shrinkage), respectively, and yellow represents slight hypertrophy (dilation).

\section{Discussion and Conclusions}

The proposed method showed better accuracy than the conventional method when the same segmentation method was used in training and testing. However, the accuracy difference became small in automated segmentation (Fig. 2), suggesting that the proposed method is more sensitive to segmentation error. As shown in Fig. 4, the proposed method appears to capture more subtle disease-specific shape deformations, which are considered to be easily affected by the segmentation error. However, among the existing methods, the segmentation method used in this study may not be the most accurate. Further, the liver segmentation accuracy may improve with future research.

The accuracy improvement by increasing the training data was larger in the proposed method than in the conventional method. One potential reason is because the healthy subspace covered a more accurate variability due to the increasing training data; thus, 
the disease subspace was less contaminated by the normal variability. When the automated segmentation is used for training, increasing the training data will be easy in the clinical routine; thus, this feature is advantageous for the proposed method.

In our dataset, the patients of the dataset belonged to the same race. Healthy livers have large inter-subject variability, but it may be smaller within the same race. To clinically accept these results, further validations, including multi-race and prospective studies, are essential. Furthermore, the fibrosis diagnosis is clinically important. In our dataset, $63 \%$ of the disease data were histologically diagnosed to be fibrotic. We tested the classifier for the dataset consisting of healthy and fibrotic livers, and obtained similar classification accuracy (0.924 for "Both-manual" and 0.889 for "Both-automated"). Thus, the proposed method will also be promising for diagnosis of fibrosis.

We have shown that the OC subspace projection pronounces the diseased components of an organ shape and more clearly clusters the healthy and diseased organs. In addition, the results showed the necessity of more accurate segmentation. Future work will include the application of the proposed method to different diagnostic problems.

Acknowledgments. This work is partly supported by KAKENHI No. 21103003.

\section{References}

1. Thompson, P., Mega, M., Woods, R., Zoumalan, C., Lindshield, C., Blanton, R., Moussai, J., Holmes, C., Cummings, J., Toga, A.: Cortical Change in Alzheimer's Disease Detected with a Disease-specific Population-based Brain Atlas. Cerebral Cortex 11(1), 1-16 (2001)

2. Heimann, T., Meinzer, H.: Statistical Shape Models for 3D Medical Image Segmentation: A review. Med. Image Anal. 13(4), 543-563 (2009)

3. Dacheng, T., Xiaoou, T.: Orthogonal Complement Component Analysis for Positive Samples in SVM Based Relevance Feedback Image Retrieval. Computer Vision and Pattern Recognition 2, 586-591 (2004)

4. Golland, P., Grimson, E., Shenton, M., Kikinis, R.: Detection and analysis of statistical differences in anatomical shape. Med. Image Anal. 9(1), 69-86 (2005)

5. Goshima, S., Kanematsu, M., Koayashi, T., Furukawa, T., Zhang, X., Fujita, H., Watanabe, H., Kondo, H., Moriyama, N., Bae, K.: Staging Hepatic Fibrosis: Computer-Aided Analysis of Hepatic Contours on Gadolinium Ethoxybenzyl Diethylenetriaminepentaacetic Acid-Enhanced Hepatocyte-Phase Magnetic Resonance Imaging. Hepatology 55(1), 328329 (2012)

6. Badeau, R., Richard, G., Bertrand, D.: Fast and stable YAST algorithm for principal and minor subspace tracking. IEEE Trans. Signal Process. 56(8), 3437-3446 (2008)

7. Higham, N.: Computing the polar decompositions-with applications. SIAM Journal on Scientific and Statistical Computing 7(4), 1160-1174 (1986)

8. Rueckert, D., Sonoda, L.I., Hayes, C., Hill, D.L.G., Leach, M.O., Hawkes, D.J.: Non-rigid registration using free-form deformations: Application to breast MR images. IEEE Trans. Med. Imaging 18(8), 712-721 (1999)

9. Okada, T., Shimada, R., Hori, M., Nakamoto, M., Chen, Y.W., Nakamura, H., Sato, Y.: Automated segmentation of the liver from 3D CT images using probabilistic atlas and multi-level statistical shape model. Acad. Radiol. 15(11), 1390-1403 (2008)

10. Ichida, F., Tsuji, T., Omata, M., Ichida, T., Inoue, K., Kamimura, T., Yamada, G., Hino, K., Yokosuka, O., Suzuki, H.: New Inuyama classification; new criteria for histological assessment of chronic hepatitis. International Hepatology Communications 6(2), 112-119 (1996) 